\title{
EGU2020-20205
}

https://doi.org/10.5194/egusphere-egu2020-20205

EGU General Assembly 2020

(c) Author(s) 2020. This work is distributed under

the Creative Commons Attribution 4.0 License.

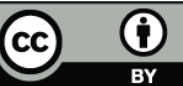

\section{Smart4RES: Towards next generation forecasting tools of renewable energy production}

George Kariniotakis ${ }^{1}$, Simon Camal ${ }^{1}$, Ricardo Bessa², Pierre Pinson ${ }^{3}$, Gregor Giebel ${ }^{3}$, Quentin Libois ${ }^{4}$, Raphaël Legrand ${ }^{4}$, Matthias Lange ${ }^{5}$, Stefan Wilbert ${ }^{6}$, Bijan Nouri ${ }^{6}$, Alexandre Neto ${ }^{7}$, Remco Verzijlbergh ${ }^{8}$, Ganesh Sauba ${ }^{9}$, George Sideratos ${ }^{10}$, Efrosyni Korka ${ }^{11}$, and Stephanie Petit

'MINES ParisTech, PSL University, Centre PERSEE, Sophia Antipolis Cedex, France (georges.kariniotakis@mines-paristech.fr, simon.camal@mines-paristech.fr)

${ }^{2}$ INESC TEC, Portugal (Rricardo.j.bessa@inesctec.pt)

${ }^{3}$ DTU, Denmark (ppin@elektro.dtu.dk, grgi@dtu.dk)

${ }^{4}$ METEO FRANCE, France (quentin.libois@meteo.fr, raphael.legrand@meteo.fr)

${ }^{5}$ Energy and Meteo Systems, Germany (matthias.lange@energymeteo.de)

${ }^{6}$ DLR, Germany (stefan.wilbert@dlr.de, bijan.nouri@dlr.de)

${ }^{7}$ EDP CNET, Portugal (alexandre.neto@edp.pt)

${ }^{8}$ WHIFFLE, Netherlands (remco.verzijlbergh@whiffle.nl)

${ }^{9}$ DNVGL, Netherlands (ganesh.sauba@dnvgl.com)

${ }^{10}$ NTUA, Greece (joesider@power.ece.ntua.gr)

11DEDDIE, Greece (e.korka@deddie.gr)

The aim of this paper is to present the objectives, research directions and first highlight results of the Smart4RES project, which was launched in November 2019, under the Horizon 2020 Framework Programme. Smart4RES is a research project that aims to bring substantial performance improvements to the whole model and value chain in renewable energy (RES) forecasting, with particular emphasis placed on optimizing synergies with storage and to support power system operation and participation in electricity markets. For that, it concentrates on a number of disruptive proposals to support ambitious objectives for the future of renewable energy forecasting. This is thought of in a context with steady increase in the quantity of data being collected and computational capabilities. And, this comes in combination with recent advances in data science and approaches to meteorological forecasting. Smart4RES concentrates on novel developments towards very high-resolution and dedicated weather forecasting solutions. It makes optimal use of varied and distributed sources of data e.g. remote sensing (sky imagers, satellites, etc), power and meteorological measurements, as well as high-resolution weather forecasts, to yield high-quality and seamless approaches to renewable energy forecasting. The project accommodates the fact that all these sources of data are distributed geographically and in terms of ownership, with current restrictions preventing sharing. Novel alternative approaches are to be developed and evaluated to reach optimal forecast accuracy in that context, including distributed and privacy-preserving learning and forecasting methods, as well as the advent of platform-enabled data-markets, with associated pricing 
strategies. Smart4RES places a strong emphasis on maximizing the value from the use of forecasts in applications through advanced decision making and optimization approaches. This also goes through approaches to streamline the definition of new forecasting products balancing the complexity of forecast information and the need of forecast users. Focus is on developing models for applications involving storage, the provision of ancillary services, as well as market participation. 